\begin{tabular}{|c|c|c|}
\hline & Int.J.Curr.Microbiol.App.Sci (2021) 10(10): 142-155 & \\
\hline EXCELLENT & $\begin{array}{l}\text { International Journal of Current Microbiology and Applied Sciences } \\
\text { ISSN: 2319-7706 Volume } 10 \text { Number } \mathbf{1 0}(\mathbf{2 0 2 1 )} \\
\text { Journal homepage: http://www.ijcmas.com }\end{array}$ & $\begin{array}{l}9 \\
39\end{array}$ \\
\hline PUBLISHERS & & www:ijcmas.com \\
\hline
\end{tabular}

\title{
Potential Application of Phage $\$ 11$ Lytic Proteins in Rapid Detection and Elimination of Staphylococcus aureus
}

\author{
Amenti and Rajkrishna Mondal* \\ Department of Biotechnology, Nagaland University, Dimapur, Nagaland, India \\ *Corresponding author
}

Keywords

Staphylococcus aureus, Phage $\phi 11$, endolysin, VAPGH, antibiotic resistant, MRSA, antimicrobials

Article Info

Accepted: 10 September 2021 Available Online: 10 October 2021

\section{A B S T R A C T}

The increasing antibiotic resistance conferred by Staphylococcus aureus to multiple potential antibiotics has become a serious issue of concern and threat to mankind worldwide. In light of this, phage lytic proteins have been reported which show potential antimicrobial activity against pathogenic microorganisms that could be a promising alternative to antibiotics to eradicate the antibiotic resistant problems. This review discusses the various applications of $S$. aureus phage lytic proteins and the potentiality of aureophage phi 11 endolysin and virion associated peptidoglycan hydrolase (VAPGH) against staphylococcus strains. Phage Phi11 endolysin harbors two enzymatically active domain; cysteine and histidine-dependent amidohydrolase/peptidase (CHAP) and Amidase 2 at the $\mathrm{N}$-terminus and a cell wall binding domain (CBD) SH3 5 at the C-terminus, while virion associated peptidoglycan hydrolase (VAPGH) has two catalytic domains, CHAP and Glucosaminidase (Mannosyl-glycoprotein endo-beta-Nacetylglucosaminidase) at its $\mathrm{N}$-terminal and C-terminal, respectively.

\section{Introduction}

Dating back to the $19^{\text {th }}$ century when phages were first discovered by the Englishman Frederick Twort in 1915 (Twort,1915) and the French-Canadian Felix d'Hérelle in 1917 (D'Herelle,1917), many countries like Russia, Georgia and Poland immediately recognized the potential antimicrobial ability of bacteriophages and phage therapy was researched and developed extensively during World War II to treat wounded soldiers and other bacterial infections until the antibiotic penicillin $G$ was successfully developed in 1942. However, over the years, due to illusage of antibiotics, antibiotic resistance exhibited by many pathogenic microorganisms such as Staphylococcus aureus, Mycobacterium tuberculosis, Bacillus anthracis, Streptococcus pneumoniae, etc., is 
a major clinical challenge worldwide. Staphylococcus aureus is distinctively one of the most important human pathogenic bacterium that is found normally in the mucous membranes and on the skin, causing minor skin infections to even more fatal disease conditions. A significant emergence of $S$. aureus strains resistant to one or combination of antibiotics, such as methicillin-resistant $S$. aureus (MRSA), vancomycin-resistant $S$. aureus (VRSA), and multiple-drug-resistant $S$. aureus (MDRSA) have been reported (Tong et al., 2015). With this concerning scenario, phage lytic proteins that are involved in the phage's lytic life cycle are being researched again envisaging that it might play a significant role in eradicating the antibiotic resistant problem. Bacteriophages are the most abundant organisms, more than any other biological entity (10-100 million per gram of soil or cc of water) on the earth that infects a bacterial cell to replicate and produce its progeny. In the beginning of its infection, it lyses the bacterial cell from outside with its tail/virion associated peptidoglycan hydrolase (VAPGHs) and at the end of their lytic cycle, its progeny virions are released from inside of the host bacterial cell by lysing the cell wall with a phage encoded protein known as endolysin.

Phage lytic proteins have one or more enzymatic domains in the $\mathrm{N}$-terminal that cleave specific peptidoglycan bond in the bacterial cell wall and can be distinguished into $N$-acetylmuramoyl-L-alanine amidase, interpeptide bridge endopeptidase, L-alanylD-glutamate endopeptidase, $\quad N$-acetyl- $\beta$-Dmuramidase, $\quad N$-acetyl- $\beta$-D-glucosaminidase depending on their specific peptidoglycan target, and a cell wall binding domain (CBD) in the C-terminal in case of endolysin. CBD is essential for recognition and binding to specific cleavage sites on the cell wall of specific bacterial strains, and also help attain its maximum catalytic activity (Schmelcher $e t$ al., 2012a). Truncated endolysin with no intact CBD showed minimum or no catalytic functions on SDS cell walls without the cell wall binding domain even in the presence of the two N-terminal catalytic domains (Sass \& Bierbaum, 2007). Staphylococcal phage lytic proteins generally have either CHAP domain or Amidase-2/3 or both domains together at the $\mathrm{N}$-terminal and the C-terminal end of endolysin contains a cell wall binding domain (CBD) that are most generally $\mathrm{SH} 35$ and SH3b in Staphylococcal phage endolysins. SH3 domains are generally small proteins $\square 60$ amino acid residues, recognition domains mostly involved in signaling pathways.

The SH3 fold consists of mainly beta-sheets arranged in a two anti-parallel $\beta$ sheets manner that lie at right angles to each other as predicted in its structure and it binds to ligands that are rich in proline motifs (P--P) (Zarrinpar et al., 2003). Staphylococcus aureus Phage $\phi 11$ is a temperate phage and it is one of the most genetically characterized $S$. aureus phages because it exhibits very high transducing efficiency and is often used to study transfer gene mutations between $S$. aureus strains. The phage genome is arranged into six functional modules: lysogeny, DNA replication, packaging, head, tail, and lysis. Virion-associated peptidoglycan hydrolases (VAPGHs) is located upstream of phage's two-component lysis module, while ORF 53 in the lytic system encodes the protein endolysin (Xia \& Wolz, 2014Among the phage encoded lytic proteins reported till date, phage phi 11 endolysin has also been identified as a potent antistaphylococcal lytic protein although no work has been done on its VAPGH so far.

This review encompasses the multi-functional ability of $S$. aureus phage lytic proteins against $S$. aureus, emphasizing mainly on $S$. aureus phage $\phi 11$ lysins as another potential candidate. 
Applications of Staphylococcus phage lytic proteins

\section{Anti-staphylococcal applications against $S$. aureus infections}

Phage encoded endolysin and virion associated cell wall hydrolase (VAPGHs) as well as chimeric lysins are gaining considerable interest as an alternative in eradicating antibiotic resistant strains to conventional antibiotics. Among the pathogenic bacteria, S. aureus is one of the most harmful human pathogenic bacteria that confer resistance to many potential antibiotics as mentioned earlier. Plenty of reports in support to phage lytic proteins as possible potential anti staphylococcal agent in vitro and in vivo have been established against $S$. aureus infections. Some of the well characterized and potent bactericidal phage encoded endolysin includes, MV-L from phage phiMR11 (Rashel et al., 2007), Lysk from phage $\mathrm{K}$ (O'Flaherty et al., 2005a; O'Flaherty et al., 2005b), PlyGRCS from phage GRCS (Linden et al., 2015), LysphiSA012 from phage phiSA012 (Fujiki et al., 2018) and LysGH15 from phage phiGH15(Gu et al., 2011a; Gu et al., 2011b; Zhang et al., 2016), have shown to exhibit efficient lytic activity against $S$. aureus strains including antibiotic resistant strains (VRSA, MRSA, VISA and teicoplanin-resistant) invitro as well as in infected mouse models. An ointment has also been formulated with LysGH15 and Apigenin (LysGH15-api-Aquaphor ointment) which showed bactericidal activity against $S$. aureus and anti-hemolysis (Cheng et al., 2018).

The modular domain structure of endolysin also provides an advantage to recombine the catalytic domain and cell wall binding domain of different phages and construct an engineered lysin that exhibits higher bacteriolytic activity, specificity, solubility, and other physicochemical nature of these enzymes which is prerequisite of a potential antimicrobial. For example, ClyS(Daniel et al., 2010), ClyH (Yang et al., 2017), ClyF (Yang et al., 2014), Ply187AN-KSH3b (Singh et al., 2014; Mao et al., 2013), these chimeric lysins have demonstrated efficient lytic activity against many antibiotic resistant $S$. aureus strains in vitro as well as in vivo. Also, a topical ointment containing ClyS has been reported to be more effective than mupirocin on a $S$. aureus infected skin (Pastagia et al., 2011).

Although, only few works have been reported on the lytic activity of $S$. aureus phages' VAGPHs against $S$. aureus, it has also shown promising effects in vitro. Some examples include, gp61 of phage MR11 (Rashel et al., 2008), truncated-fusion and whole $\mathrm{HydH} 5$, which have shown efficient lysis of live $S$. aureus cells and $S$. aureus $\mathrm{Sa} 9$ cells, bovine and human $S$. aureus strains, MRSA strain N315, and human Staphylococcus epidermidis strains in three different studies by Rodriguez et al., (Rodríguez et al., 2011; Rodríguez et al., 2012; Rodríguez et al., 2013). Also, some other chimeric peptidoglycan hydrolases against $S$. aureus strains such as; P16-17 of phage P68 (Manoharadas et al., 2009; Takác and Blas, 2005) and P128 of phage $\mathrm{K}$ against global clinical isolates of $S$. aureus including drug resistant strains in vitro as well as in vivo (Sundarrajan et al., 2014; Channabasappa et al., 2018; Paul et al., 2011) have been reported.

\section{Application in food safety}

Food-borne diseases in humans cause by $S$. aureus and its derived toxins are also a concerning global issue and therefore its detection and eradication in foods and biofilm destruction on food surfaces and utensils is an emergent need. Purified lytic proteins have been demonstrated as a safe and stable 
additive into food products to control the contamination of food borne pathogens. For example, LysH5 have shown rapid lysis of $S$. aureus present in pasteurized milk after $4 \mathrm{~h}$ of incubation at $37^{\circ} \mathrm{C}$ (Obeso et al., 2008) and also biofilm removal. Purified LysSA97 in synergy with carvacrol have shown anti staphylococcal activity in skimmed milk, whole milk and lean beef (Chang et al., 2016). Purified LysSA11 endolysin have also shown significant reduction in MRSA colony in milk and ham as well as on the surfaces of polypropylene plastic cutting boards and stainless-steel knives (Chang et al., 2017). Three lytic proteins (HydH5Lyso, HydH5SH3b, CHAPSH3b) constructed by fusion of different domains of Staphylococcus aureus phage vB_SauS-phiIPLA88 VAPGH HydH5 and lysostaphin have also demonstrated antimicrobial activity against $S$. aureus strain $\mathrm{Sa} 9$ in milk and extension of commercial whole milk shelf life (Rodríguez et al., 2013).

\section{Synergistic application of phage lytic proteins with antibiotics}

Potential synergistic ability of endolysin can be achieved by combination with VAPGHs or other phage's endolysin or antibiotics. This synergism can lead to improved lytic efficacy, requirement of low antimicrobial dosage and reduces resistance by targeting different multiple cleavage sites or the cleavage of a particular site by the first catalytic domain which indirectly enhances the lytic activity of the conjugated endolysin catalytic domain or VAGPHs or antibiotic. Becker et al., (2008) have reported the synergistic effects observed in vitro of $S$. aureus endolysin LysK CHAP domain with lysostaphin glycylglycine endopeptidase (Becker et al., 2008). Schmelcher et al., (2012b) have also investigated the synergistic effect of two chimeric proteins of the streptococcal $\lambda \mathrm{SA} 2$ endolysin endopeptidase domain conjugated with cell wall binding domains of lysostaphin and LysK and both the constructs showed lysis of 16 different $S$. aureus mastitis isolates, including penicillin-resistant strains in vitro and in vivo in mouse model (Schmelcher et al., 2012b). Endolysin MV-L from S. aureus phage phi MR11 have also showed synergistic staphylocidal effect with glycopeptide antibiotics vancomycin and teicoplanin against VISA and prevention from MRSA septic death by intraperitoneal administration of MV-L without any observed side effects (Rashel et al., 2007). Chimeric staphylococcal endolysin ClyS have also shown synergistic effect in combination with vancomycin and oxacillin by protecting mice from MRSA induced septicemia (Daniel et al., 2010). Also, LysH5 endolysin encoded by the staphylococcal bacteriophage phi-SauSIPLA88, in combination with nisin increased the lytic activity of LysH5 by 8-fold and showed a strong synergistic effect in eliminating $S$. aureus Sa9 present in pasteurized milk (Garcia et al., 2010).

\section{Application in detection of $S$. aureus strains}

S. aureus infections are mostly mediated by the production of its virulence factors such as staphylococcal exotoxins (SEs), among which SEA and SEB are most commonly found and characterized for pathogen detection (Pinchuk et al., 2010). Systematic and rapid detection of the bacterium or its toxins is a critical step in order to eradicate the pathogen efficiently. Wu et al., (2016) have also reviewed different types of conventional and modern techniques, and its limitations for detection of $S$. aureus toxins. Early conventional methods include direct tests on animal models which involves negative ethical issues. More reliable, rapid, sensitive and specific methods which include, antigen-antibody binding based serological tests such as gel diffusion, agglutination assays and ELISA, and immunoassays coupled with sensitive detecting elements; 
molecular biological techniques such as specific hybridization and PCR based methods and chromatographic techniques, have been developed, however, it requires highly stable antibodies, expensive and complicated instruments, detection elements and PCR machines as well as skilled trained professionals to handle.

Recently, aptamer (short ssDNA or RNA and peptides) based biosensors proves to be a better alternative to counter the associated limitations with traditional techniques and assays which employ antigen/antibody based for microbial detection. Aptamers generally are shorter and possess smaller molecular weight than antibodies which make them superior in stability. It can be easily synthesized in vitro and also allows flexible modification with various chemical tags such as fluorophores and nanoparticles that do not interfere with efficient target recognition (Wu et al., 2016). Phage lytic proteins can be modeled as short aptamers, owing to its structure characterized by different functional domains that can be reconstructed. The Cterminal cell wall binding domain binds specifically to its host bacteria's peptidoglycan layer as reported in many studies. This specific affinity of endolysin CBDs to bacterial cell walls can be exploited in different ways to detect infectious bacteria in clinical and food safety as shown in Fig. 1.

One of the simplest and mostly reported method is fluorescent microscopic detection of target pathogens by phage endolysin CBDs tagged with fluorescent proteins, which can be detected upon specific binding to targeted cell walls. Gu et al., (2011c) have reported the specific binding of a fusion endolysin protein, LysGH15B with green fluorescent protein (LysGH15B-GFP) to staphylococcal isolates, including MRSA, while the unbound GFP did not display any fluorescence when scanned under laser scanning confocal microscope $(\mathrm{Gu}$ et al., 2011c). Listeria strains from a mixed bacterial culture were rapidly differentiated by conjugating listeria infecting phage endolysin with fluorescent markers (Schmelcher et al., 2010). Loessner et al., (2002) have also demonstrated specific binding of two Listeria monocytogenes phage endolysins Ply118 and Ply500 CBDs fused with GFP to listeria cells of different serovar groups (Loessner et al., 2002). Similarly, The CBD domain from a streptococcal phage was fused with GFP which showed that the recombinant CBD was able to bind to living cells of Staphylococcus aureus and Streptococcus agalactiae (Jarabkova et al., 2020).

Secondly, surface immobilization of target cells on magnetic beads or magnetic nanoparticles coated with CBDs can be employed for efficient recovery and detection of pathogens in food, water and blood samples. Chibli et al., (2014) have exploited the specific binding ability of phage endolysins LysK and phi11, and the bacteriocin lysostaphin, coated on silicon wafers to detect staphylococcal strains wherein it specifically binds to eight tested clinical isolates of $S$. aureus and $S$. epidermidis (Chibli et al., 2014).

Paramagnetic beads coated with recombinant Listeria phage endolysin-derived CBDs have also shown efficient and sensitive detection than conventional standard procedures of $L$. monocytogenes cells from suspended cultures as well as artificially and naturally contaminated food samples (Kretzer et al., 2007). Similarly, a novel strategy has been developed by Yi et al., (2019) for sensitive detection of $S$. aureus, which employs PlyV12 CBD-functionalized magnetic beads for separation and detection by fluorescence system of horseradish peroxidase (HRP), hydrogen peroxide and Amplex Red (Yi et al., 2019). Park et al., (2018) performed an ATP bioluminescence assay for $B$. cereus detection 
using CBD-conjugated magnetic nanoparticles (CBD-MNPs) which showed sensitive detection up to as few as $10 \mathrm{CFU} / \mathrm{ml}$ and 103 $\mathrm{CFU} / \mathrm{ml}$ in buffer and blood, with no crossreactivity with other microflora (Park et al., 2018).

Conjugation of CBDs to nanoparticles is another feasible method which can be exploited in many pathogen detection assays. Nanoparticles can be of different types such as magnetic nanoparticles, fluorescent nanoparticles and metallic nanoparticles, for example, gold or silver nanoparticles, which are advantageous owing to their large surface area for immobilization of different molecules that can bind specifically to their target molecules, and also the ability to emit signals upon binding. Kong et al., (2017) have developed a nitrocellulose-based lateral flow assay employing CBD conjugated colloidal gold nanoparticle for the detection of Bacillus cereus. The result showed sensitivity up to $1 \times 104 \mathrm{CFU} / \mathrm{ml}$ in 20 minutes and superior to conventional antibody-antigen based assay (Kong et al., 2017). Kim et al., (2018) have also demonstrated the selective killing of Bacillus anthracis from a mixed culture including B. subtilis and S. aureus by silver nanoparticle conjugated with fluorescent protein tagged-CBD of $B$. anthracis (Kim et al., 2018).

Staphylococcus aureus phage phi 11 lytic proteins

Till date, few studies have been reported only on $S$. aureus phage $\phi 11$ endolysin as a potent anti-staphylococcal lysin. Navarre et al., (1999) first reported the lytic activity of its $\mathrm{N}$ terminal catalytic domains, CHAP and Amidase 3 on Staphylococcal cell wall and solubilized surface protein from staphylococcal cell wall (Navarre et al., 1999). Phi 11 endolysin has also shown potential in vitro lysis and anti-biofilm activity against a number of staphylococcal strains including $S$. epidermidis and $S$. simulans, cell surface mutants and MRSA by whole endolysin as well as truncated parental endolysin as efficient as lysostaphin. Also, in vivo treatment of systemic MRSA infected mouse models with phi 11 endolysin, showed $100 \%$ protection from death (Schmelcher et al., 2015; Sass \& Bierbaum, 2007). In another study, the whole phi 11 endolysin and truncated part that possessed only its CHAP domain have also shown great anti staphylococcal activity against live whole cells of mastitis pathogens, Staphylococcus aureus and coagulase-negative staphylococci (Staphylococcus chromogenes, Staphylococcus epidermidis, Staphylococcus hyicus, Staphylococcus simulans, Staphylococcus warneri and Staphylococcus xylosus) as well as in contaminated milk (Donovan et al., 2006).

\section{Staphylococcus aureus bacteriophage $\phi 11$ endolysins}

Phage Phi 11 endolysin is 481 amino acid residues long encoded by ORF 53, with GenBank accession no. AAL82281.2. It possesses two enzymatically active domains (EAD); CHAP (D-alanyl-glycyl endopeptidase) and Amidase 2 (Nacetylmuramyl-L-alanine amidase) at its $\mathrm{N}$ terminal and a CBD (cell wall binding domain) at its C-terminal site (Fig 2a), as predicted by PFAM 33.1 server (El-Gebali et al., 2019). Phi 11 endolysin cleaves at two sites of $S$. aureus peptidoglycan layer, the DAla-Gly peptide bond by CHAP and the $N$ acetylmuramyl-L-Ala amide bond by Amidase-2 as suggested by Navarre, et al., (1999). The C-terminal cell wall binding site belongs to SH3 5 (SRC Homology) CBD group that bears close homology to the cell wall targeting domain of lysostaphin (Navarre et al., 1999). 
Fig.1 Schematic diagram showing different phage endolysin cell wall binding domain's conjugates for efficient pathogen detection

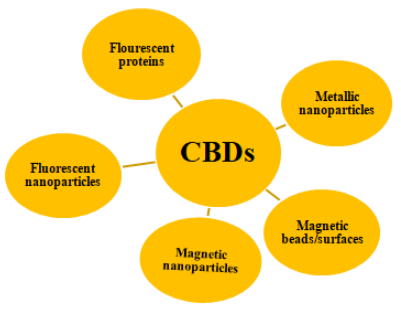

Fig.2 Conserved domain analysis by PFAM 33.1. (a) Three domains recognized on Phi 11 endolysin; CHAP (20 to 113 amino acids), Amidase 2 (196 to 324 amino acids) and SH3 5 CBD (395 to 460 amino acids). (b) Two domains identified on Phi 11 VAPGH; CHAP (34 to 120 amino acids) and Glucosaminidase (494 to 616 amino acids)

a)

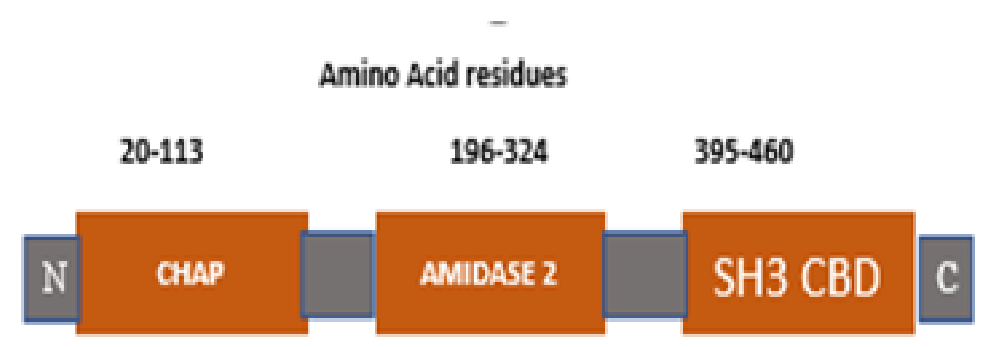

b)

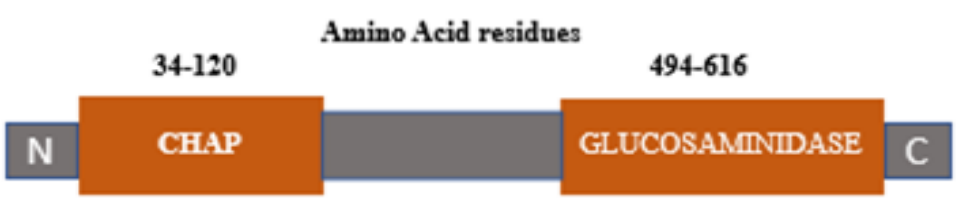


Fig.3 Multiple sequence alignment by CLUSTAL OMEGA; amino acid residues conserved between the aligned protein sequences are highlighted by black (100\%) and grey (up to 50\%). (a)

Phi 11 endolysin aligned with other seven reported endolysins from S. aureus phages; LysK

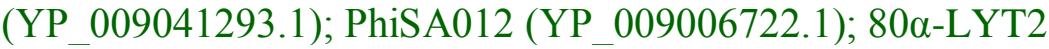

YP_001285385.1);Twort(YP_238716.1); MV-L (YP_001604156.1); LysH5 (ACE77796.1); and GH15 (ADG26756.1). (b) Phi 11 cell wall hydrolase (VAPGH) aligned with other three reported VAPGHs from S. aureus phages; GP61 (ACJ64589.1); HydH5 (ACJ64586); and 80 $\alpha$ (YP_001285381.1).

(a)

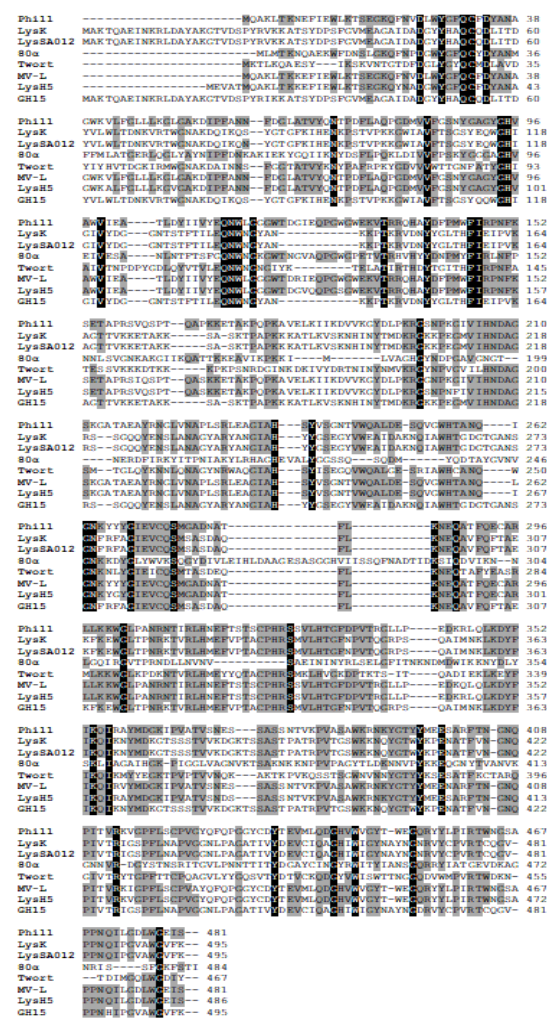


(b)

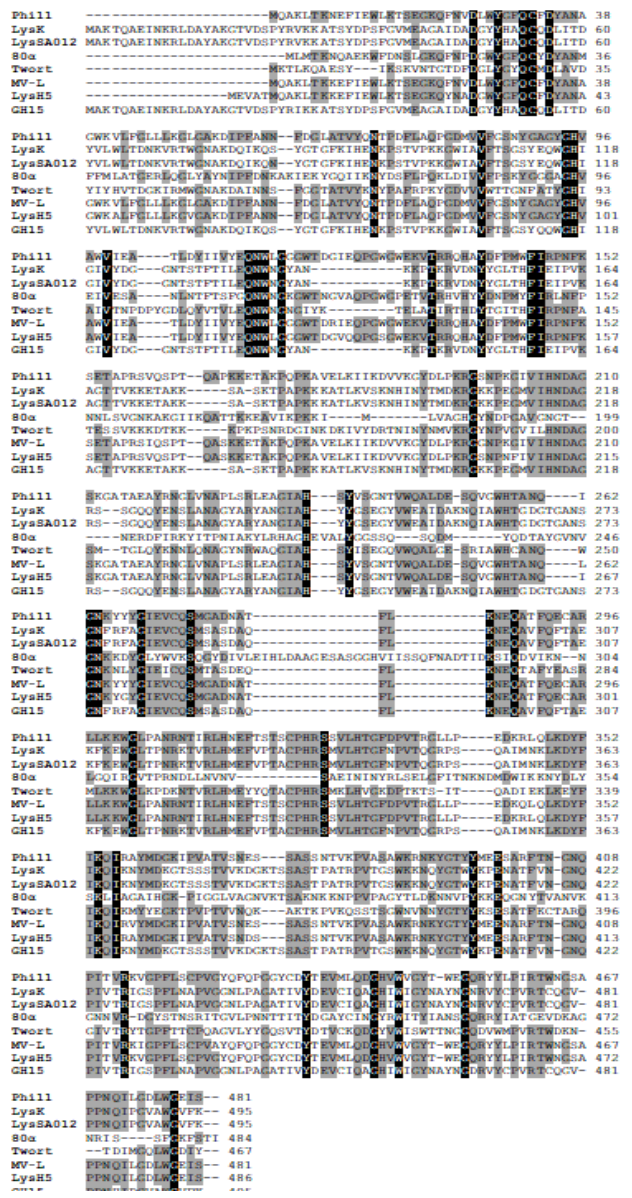

Sequence alignment with seven other well characterized $S$. aureus phage endolysin using CLUSTAL OMEGA software (Madeira et al., 2019), showed significant sequence similarity with MV-L and LysH5 (97.7 \% and 96.1\% respectively) (Fig 3a). The stability of the recombinant phi11 endolysin at different storage $\left(4^{\circ} \mathrm{C}\right.$ and $\left.22^{\circ} \mathrm{C}\right)$ and working temperatures $\left(37^{\circ} \mathrm{C}\right)$ and $\mathrm{pH}$ level have also been studied. They have stated that secondary structures of the protein play the primary role in all the factors determining its stability. From the CD spectra of secondary structures, it has observed a sharp rise in the number of random coils $(26.8 \pm 4.2 \%$ to $43.1 \pm 4.0 \%)$, with increasing temperature and $\mathrm{pH}$. The report showed that an increase in $\mathrm{pH}$ from 7.5 to alkaline range (up to 9) and temperature from $4{ }^{\circ} \mathrm{C}$ to $22^{\circ} \mathrm{C}$ to $37^{\circ} \mathrm{C}$, the half inactivation time of the protein decreased from 140 days to 8 days to 10 mins, respectively (Filatova et al., 2016).

\section{Staphylococcus aureus phage phi 11 virion associated peptidoglycan hydrolase (VAPGH)}

Phi 11 VAPGH has 632 amino acid residues (GenBank accession no. AAL82277.1) constituted of only two EADs (CHAP and Glucosaminidase) and no $\mathrm{CBD}$ (Fig 2b), as predicted by PFAM 33.1 server (El-Gebali et al., 2019). Sequence alignment with other three reported S. aureus phage VAPGHs using CLUSTAL OMEGA software (Madeira et al., 2019), have shown a significant sequence similarity with $80 \alpha$ and HydH5 (98.4 \% and $90.1 \%$ respectively) followed by G61 which 
shows only $57 \%$ similarity (Fig 3b). Although no work has been done on the cell lysis potency of phi 11 VAPGH, it shows significant sequence homology to HydH5, which have been well characterized and reported as a potent staphylococcal lytic protein which is also highly thermostable, retaining its activity up to $72 \%$ at $100^{\circ} \mathrm{C}$ (Rodriguez et al., 2011).

The whole idea of lytic protein as an antibacterial is that it should be able to efficiently lyse the bacteria from outside when applied exogenously, which gives a bigger prospective to VAPGH considering its natural mode of action that lyse a bacterial cell from outside against different physiological and environmental conditions in contrast to the activity of phage encoded endolysin, which lyse the cell from within in a closed defined system that in most cases after holin-mediated cell membrane disruption.

Bacteriophages and its lytic proteins may be the new frontier in combating infectious and antibiotic resistant pathogenic bacteria. Notable progression has been made in recognition of phage lytic proteins as prospective antibacterial agents against $S$. aureus, however therapeutically it is still objectionable in most cases and some limitations associated particularly with systemic application still need to be addressed, which includes, stability (half-life inactivation time), immunogenicity, toxicity and efficient penetration into specific cell or tissues. Many potential aspects of bacterial phage lytic proteins as a whole or as engineered chimeric lysins have been discussed in this review. The potentiality of phi 11 endolysin have been reported by few studies although not much work has been done with phi 11 VAPGH, more distinctively on its lytic activity. As discussed earlier, VAPGH lyses a bacterial cell from outside during the initial infection cycle against different environmental constraints and specifically even without the presence of a cell wall binding domain. Considering all these propensities of a VAPGH and its significant homology with HydH5, phi 11 VAPGH can also be a potential prospect against $S$. aureus strains, including antibiotic resistant strains.

\section{Acknowledgments}

The authors gratefully acknowledge the logistic support of Department of Biotechnology, Nagaland University, India. Ms. Amenti is also thankful to Department of Biotechnology, Government of India for providing regular monthly Research Scholarship.

\section{References}

Becker, S. C., Foster-Frey, J., \& Donovan, D. M. (2008). The phage K lytic enzyme LysK and lysostaphin act synergistically to kill MRSA. FEMS Microbiology Letter 287(2),185-191.

Chang, Y., Kim, M., \& Ryu, S. (2107). Characterization of a novel endolysin LysSA11 and its utility as a potent biocontrol agent against Staphylococcus aureus on food and utensils. Food Microbiology, 68:112-120.

Chang, Y., Yoon, H., Kang, D. H., Chang, P.S.,\& Ryu, S. (2016). Endolysin LysSA97 is synergistic with carvacrol in controlling Staphylococcus aureus in foods. International Journal of Food Microbiology,244,19-26.

Channabasappa, S., Chikkamadaiah, R., Durgaiah, M., Kumar, S., Ramesh, K., Sreekanthan, A., \& Sriram, B. (2018). Efficacy of chimeric ectolysin P128 in drug-resistant Staphylococcus aureus bacteremia in mice. Journal of Antimicrobial Chemotherapy, 73(12), 3398-3404.

Cheng, M., Zhang, L., Zhang, H., Li, X., Wang, Y., Xia, F., Wang, B., Cai, R., Guo, Z., Zhang, Y., Ji, Y., Sun, C., Feng, X., Lei, 
L., Yang, Y., Han, W., \& Gu, J. (2018). An Ointment Consisting of the Phage Lysin LysGH15 and Apigenin for Decolonization of Methicillin-Resistant Staphylococcus aureus from Skin Wounds. Viruses, 10(5):244.

Chibli, H., Ghali, H., Park, S., Peter, Y. A., \& Nadeau, J. L. (2014). Immobilized phage proteins for specific detection of staphylococci.Analyst, 139, 179- 186.

Daniel, A., Euler, C., Collin, M., Chahales, P., Gorelick, K. J., \& Fischetti, V. A. (2010). Synergism between a novel chimeric lysin and oxacillin protects against infection by methicillin-resistant Staphylococcus aureus. Antimicrobial Agents and Chemotherapy, 54, 1603-1612.

D'Herelle, F. (1917). An invisible microbe that is antagonistic to the dysentery bacillus. ComptesRendus(in French), 165, 373-75.

Donovan, D. M., Lardeo, M., \& Foster-Frey, J. (2006). Lysis of staphylococcal mastitis pathogens by bacteriophage phil1 endolysin. FEMS Microbiology Letter, 265, 133-139.

El-Gebali, S., Mistry, J., Bateman, A., Eddy, S. R., Luciani, A., Potter, S. C., Qureshi, M., Richardson, L. J., Salazar, G. A., Smart, A., Sonnhammer, E. L. L., Hirsh, L., Paladin, L., Piovesan, D., Tosatto, S. C. E., \& Finn, R. D. (2019). The Pfam protein families database in 2019. Nucleic Acids Research, 47(D1), D427-D432

Filatova, L. Y., Donovan, D. M., Foster-Frey, J. A., Pugachev, V. G., Kudryashova, E. V., \& Klyachko, N. L. (2016). Lytic enzymes of staphylococcal phages: Correlation between secondary structure and stability. Moscow University Chemistry Bulletin, Vol. 71, No. 1, pp. 7-11.DOI: 10.3103/S0027131414030043.

Fujiki, J., Nakamura, T., Furusawa, T., Ohno, H., Takahashi, H., Kitana, J., Usui, M., Higuchi, H., Tanji, Y., Tamura, Y., \& Iwano, H. (2018). Characterization of the lytic capability of a LysK-like endolysin, Lys-phiSA012, derived from a polyvalent Staphylococcus aureus bacteriophage. Pharmaceuticals (Basel), 11(1), 25.
Garcia, P., Martinez, B., Rodriguez, L., \& Rodriguez, A. (2010). Synergy between the phage endolysin LysH5 and nisin to kill Staphylococcus aureus in pasteurized milk. International Journal of Food Microbiology, 141, 151-155.

Gu, J., Lu, R., Liu, X., Han, W., Lei, L., Gao, Y., Zhao, H., Li, Y., \& Diao, Y. (2011c). LysGH15B, the SH3b domain of staphylococcal phage endolysin LysGH15, retains high affinity to staphylococci. Current Microbiology, 63(6), 538-542.

Gu, J., Xu, W., Lei, L., Huang, J., Feng, X., Sun, C., Du, C., Zuo, J., Li, Y., Du, T., Li, L., \& Wenyu Han, W. (2011a). LysGH15, a novel bacteriophage lysin, protects a murine bacteremia model efficiently against lethal methicillin-resistant Staphylococcus aureus infection. Journal of Clinical Microbiology, 49(1),111-7.

Gu, J., Zuo, J., Lei, L., Zhao, H., Sun, C., Feng, X., Du, C., Li, X., Yang, Y., \& Wenyu Han, W. (2011b). LysGH15 reduces the inflammation caused by lethal methicillin-resistant Staphylococcus aureus infection in mice. Bioengineered bugs,2(2):96-9.

Jarabkova, V., Tisakova, L., Benešík, M., \& Godány, A. (2020). SH3 binding domains from phage endolysins: how to use them for detection of gram-positive pathogens. Journal of Microbiology, Biotechnology and Food Sciences, 9 (6), 1215-1220.

Kim, D. Kwon, S. J., Wu, X., Sauve, J., Lee, I., Nam, J., Kim, J., \& Dordck, J. S. (2018). Selective killing of pathogenic bacteria by antimicrobial silver nanoparticle-cell wall binding domain conjugates. ACS Applied Materials \& Interfaces, 10, 13317-13324.

Kong, M., Shin, J. H., Heu, S., Park, J. K., \& Ryu, S. (2017). Lateral flow assay based bacterial detection using engineered cell wall binding domains of a phage endolysin. Biosensors and Bioelectronics, 96, 173-177.

Kretzer, J. W., Lehmann, R., Schmelcher, M., Banz, M., Kim, K. P., Korn, C., \& Loessner, M. J. (2007). Use of high- 
affinity cell wall-binding domains of bacteriophage endolysins for immobilization and separation of bacterial cells. Applied Environmental Microbiology, 73, 1992-2000.

Linden, S. B., Zhang, H., Heselpoth, R. D., Shen, Y., Schmelcher, M., Eichenseher, F., \& Nelson, D. C. (2015) Biochemical and biophysical characterization of PlyGRCS, a bacteriophage endolysin active against methicillin-resistant Staphylococcus aureus. Applied Microbiology and Biotechnology, 99(2), 741-52.

Loessner, M. J., Kramer, K., Ebel, F., \& Scherer, S. (2002).C-terminal domains of Listeria monocytogenes bacteriophage murein hydrolases determine specific recognition and high-affinity binding to bacterial cell wall carbohydrates. Molecular Microbiology, 44(2), 335-349.

Madeira, F., Park, Y. M., Lee, J., Buso, N., Gur, T., Madhusoodanan, N., Basutkar, P., Tivey, A. R. N., Potter, S. C., Finn, R. D., \& Lopez, R. (2019). The EMBL-EBI search and sequence analysis tools APIs in 2019. Nucleic Acids Research, 47(W1), W636-W641.

Manoharadas, S., Witte, A., \& Bläsi, U. (2009) Antimicrobial activity of a chimeric enzybiotic towards Staphylococcus aureus. Journal of Biotechnology, 139(1):118-23

Mao, J., Schmelcher, M., Harty, W. J., FosterFrey, J., \& Donovan, D. M. (2013). Chimeric Ply187 endolysin kills Staphylococcus aureus more effectively than the parental enzyme. FEMS Microbiology Letters, 342,30-6.

Navarre, W. W., Ton-That, H., Faull, K. F., \& Schneewind, O. (1999). Multiple enzymatic activities of the murein hydrolase from staphylococcal phage phi11. Identification of a D-alanyl-glycine endopeptidase activity. Journal of Biological Chemistry. 274, 15847-15856.

O'Flaherty, S., Coffey, A., Meaney, W., Fitzgerald, G., \& Ross, R. (2005a). The recombinant phage lysin LysK has a broad spectrum of lytic activity against clinically relevant staphylococci, including methicillin-resistant

Staphylococcus aureus. Journal of Bacteriology, 187, 7161-7164.

O'Flaherty, S., Ross, R., Meaney, W., Fitzgerald, G., Elbreki, M., \& Coffey, A. (2005b). Potential of the polyvalent antiStaphylococcus bacteriophage $\mathrm{K}$ for control of antibiotic-resistant staphylococci from hospitals. Applied Environmental Microbiology, 71, 18361842.

Obeso, J. M., Martínez, B., Rodríguez, A., \& García, P. (2008). Lytic activity of the recombinant staphylococcal bacteriophage PhiH5 endolysin active against Staphylococcus aureus in milk. International Journal of Food Microbiology, 128(2), 212-8

Park, C., Kong, M., Lee, J. H., Ryu, S., \& Park, S. (2018). Detection of Bacillus Cereus Using Bioluminescence Assay with Cell Wall-binding Domain Conjugated Magnetic Nanoparticles. BioChip Journal, 12(4), 287-293.

Pastagia, M., Euler, C., Chahales, P., FuentesDuculan, J., Krueger, J. G., \& Fischetti, V. A. (2011). A novel chimeric lysin shows superiority to mupirocin for skin decolonization of methicillin-resistant and -sensitive Staphylococcus aureus strains. Antimicrobial Agents and Chemotherapy, 55, $738-744$.

Paul, V. D., Rajagopalan, S. S., Sundarrajan, S., George, S. E., Asrani, J. Y., Pillai, R., Chikkamadaiah, R., Durgaiah, M., Sriram, B., \& Padmanabhan, S. (2011).A novel bacteriophage Tail-Associated Muralytic Enzyme (TAME) from Phage $\mathrm{K}$ and its development into a potent antistaphylococcal protein. $B M C$ Microbiology, 11, 226.

Pinchuk, I. V., Beswick, E. J., \& Reyes, V.E. (2010). Staphylococcal enterotoxins. Toxins 2, 2177-2197.

Rashel, M., Uchiyama, J., Takemura, I., Hoshiba, H., Ujihara, T., Takatsuji, H., Honke, K., \& Matsuzaki, S.(2008). Tail- 
associated structural proteingp61 of Staphylococcus aureus phage phi MR11 has bifunctionallytic activity. FEMS Microbiology Letters, 284, 9-16.

Rashel, M., Uchiyama, J., Ujihara, T., Uehara, Y., Kuramoto, S., Sugihara, S., Yagyu, KI., Muraoka, A., Sugai, M., \& Hiramatsu, K. (2007). Efficient elimination of multidrug-resistant Staphylococcus aureus by cloned lysin derived from bacteriophage $\phi \mathrm{MR} 11$. The Journal of Infectious Diseases, 196, 1237-1247.

Rashel, M., Uchiyama, J., Ujihara, T., Uehara, Y., Kuramoto, S., Sugihara, S., Yagyu, K., Muraoka, A., Sugai, M., Hiramatsu, K., Honke, K., \& Matsuzaki, S. (2007). Efficient elimination of multidrugresistant Staphylococcus aureus by cloned lysin derived from bacteriophage phi MR11. The Journal of Infectious Diseases, 196(8),1237-47.

Rodríguez, L., Martínez, B., Zhou, Y., Rodríguez, A., Donovan, D. M., \& García, P. (2011). Lytic activity of the virion-associated peptidoglycanhydrolase HydH5 of Staphylococcus aureus bacteriophage vB_SauS-phiIPLA88. BMC Microbiology, 11, 138.

Rodríguez-Rubio, L., Martínez, B., Donovan, D. M., García, P.,\& Rodríguez, A. (2013). Potential of the Virion-Associated Peptidoglycan Hydrolase HydH5 and its derivative fusion proteins in milk bio preservation. PLOS One, 8(1), e54828.

Rodríguez-Rubio, L., Martínez, B., Rodríguez, A., Donovan, D. M., \& García P. (2012). Enhanced staphylolytic activity of the Staphylococcus aureus bacteriophage vB_SauS-phiIPLA88 HydH5 virion associated peptidoglycan hydrolase: fusions, deletions, and synergy with LysH5. Applied Microbiology and Biotechnology, 78, 2241-2248.

Sass, P., \& Bierbaum, G. (2007). Lytic activity of recombinant bacteriophage phil1 and phi12 endolysins on whole cells and biofilms of Staphylococcus aureus. Applied Environmental Microbiology,73(1), 347-52.
Sawyer, S., \& Tapia, A. (2005). The sociotechnical nature of mobile computing work: Evidence from a study of policing in the United States. International Journal of Technology and Human Interaction, 1(3), 1-14.

Schmelcher, M., Donovan, D.M., \& Loessner, M. J. (2012a). Bacteriophage endolysins as novel antimicrobials. Future Microbiology, 7(10), 1147-71.

Schmelcher, M., Powell, A. M., Becker, S. C., Camp, M. J., \& Donovan, D. M. (2012b). Chimeric phage lysin act synergistically with lysostaphin to kill mastitis-causing Staphylococcus aureus in murine mammary glands. Applied Environmental Microbiology, 78(7), 2297-2305.

Schmelcher, M., Shabarova, T., Eugster, M. R., Eichenseher, F., Tchang, V. S., Banz, M., \& Loessner, M. J. (2010). Rapid multiplex detection and differentiation of Listeria cells by use of fluorescent phage endolysin cell wall binding domains. Applied Environmental Microbiology, 76(17), 5745-5756.

Schmelcher, M., Shen, Y., Nelson, D. C., Eugster, M. R., Eichenseher, F., Hanke, D. C., Loessner, M. J., Dong, S., Pritchard, D. G., \& Lee, J. C. (2015). Evolutionarily distinct bacteriophage endolysins featuring conserved peptidoglycan cleavage sites protect mice from MRSA infection. Journal of Antimicrobial Chemotherapy, 70, 14531465.

Singh, P. K., Donovan, D. M., \& Kumar, A. (2014). Intravitreal injection of the chimeric phage endolysin Ply187 protects mice from Staphylococcus aureus endophthalmitis. Antimicrobial Agents and Chemotherapy, 58, 4621-9.

Sundarrajan, S., Raghupatil, J., Vipra, A., Narasimhaswamy, N., Saravanan, S., Appaiah, C., Poonacha, N., Desai, S., Nair, S., Bhatt, R.N., Roy, P., Chikkamadaiah, R., Durgaiah, M., Sriram, B., Padmanabhan, S., \& Sharma, U. (2014). Bacteriophage-derived CHAP domain protein, P128, kills 
Staphylococcus cells by cleaving interpeptide cross-bridge of peptidoglycan. Microbiology Society, 160(Pt 10):2157-2169.

Takác, M., \& Bläsi, U. (2005). Phage P68 virion-associated protein 17 displays activity against clinical isolates of Staphylococcus aureus. Antimicrobial Agents and Chemotherapy, 49(7), 293440.

Tong, S. Y., Davis, J. S., Eichenberger, E., Holland, T. L., \& Fowler, V. G. (2015). Staphylococcus aureus infections: epidemiology, pathophysiology, clinical manifestations, and management. Clinical Microbiology Reviews, 28(3), 603-61.

Twort, F. W. (1915). An investigation on the nature of ultra-microscopic viruses. The Lancet, 186 (4814), 1241-43.

Wu, S., Duan, N., Gu, H., Hao, L., Ye, H., Gong, W., \& Wang, Z. (2016). A Review of the Methods for Detection of Staphylococcus aureus Enterotoxins. Toxins, 8(7), 176.

Xia, G., \& Wolz, C. (2014). Phages of Staphylococcus aureus and their impact on host evolution infection. Infection, Genetics and Evolution, 21, 593-601

Yang, H., Zhang, H., Wang, J., Yu, J., \& Wei, H. (2017). A novel chimeric lysin with robust antibacterial activity against planktonic and biofilm methicillin resistant Staphylococcus aureus. Scientific Reports, 7, 40182.

Yang, H., Zhang, Y., Yu, J., Huang, Y., Zhang, X. E., \& Wei, H. (2014). Novel chimeric lysin with high-level antimicrobial activity against methicillin-resistant Staphylococcus aureus in vitro and in vivo. Antimicrobial Agents and Chemotherapy, 58, 536-542.

Yi, Z., Wang, S., Meng, X., Wu, A., Li, Q., Song, Y., Zhao, R., \& Qiao, J. (2019). Lysin cell-binding domain-functionalized magnetic beads for detection of Staphylococcus aureus via inhibition of fluorescence of Amplex red/hydrogen peroxide assay by intracellular catalase. Analytical and Bioanalytical Chemistry, 411, 7177-7185.

Zarrinpar, A., Bhattacharyya, R. P., \& Lim, W. A. (2003) The structure and function of proline recognition domains. Science Signaling,

DOI: 10.1126/stke.2003.179.re8

Zhang, L., Li, D., Li, X. W., Hu, L. Y., Cheng, M. J., Xia, F. F., Gong, P. J., Wang, B., Ge, J. L., \& Zhang, H. (2016). LysGH15 kills Staphylococcus aureus without being affected by the humoral immune response or inducing inflammation. Scientific Reports, 6, 29344.

\section{How to cite this article:}

Amenti and Rajkrishna Mondal. 2021. Potential Application of Phage $\phi 11$ Lytic Proteins in Rapid Detection and Elimination of Staphylococcus aureus. Int.J.Curr.Microbiol.App.Sci. 10(10): 142-155. doi: https://doi.org/10.20546/ijcmas.2021.1010.017 